\title{
Early effects of the Covid-19 on the Agricultural field: Evidence from two major commodities
}

\author{
Theodoros Daglis \\ National Technical Univeristy of Athens
}

\begin{abstract}
:
The Covid-19 pandemic has changed the dynamics of the overall economy, impacting many fields. Such a case is the agricultural sector, which was impacted in a significant manner. In this paper, we examine two most important commodities from the agricultural sector, namely the Oats and the Wheat, during the Covid-19 spread and the lockdown measures. Using relevant time series specifications, we establish a hypothesis regarding the effect of the Covid-19 pandemic on the values of these two commodities. Based on our findings, the values of the commodities were affected by the Covid-19 spread and moreover, the Covid-19 spread provides useful information for the predicting and forecasting of these values. Our findings are robust, since the out-of-sample forecasting accuracy of the alternative model employed, that explicitly incorporate the pandemic induced by the SARS-COV-2 virus, is superior to the baseline model.
\end{abstract}

Keywords: COVID-19, Agriculture, Oats, Wheat

JEL codes: C22, C58, C50, C51

\section{Introduction}

The Covid-19 pandemic has changed the dynamics of the overall economy, impacting many fields. Such a case is the agricultural sector, as the fear spread by the Covid-19, has led to the excess demand from the market, of the major commodities of the primary sector. The lockdown measures made the matters worse, as the fear of a long-term quarantine had an impact to the consumers. Furthermore, government organizations posed interruptions in the acquirement of nourishment grains. This includes lockdown measures, panic spread, leading to lack of laborers to collect the crops, deficiency of drivers in the transportation area, disturbances in the assortment of harvests from the homesteads by private dealers (Ganesan and Micheal, 2020). Moreover, the export restrictions and other trade policy measures, following the COVID-19 crisis made matters 
2nd International Conference on Applied Research in BUSINESS, MANAGEMENT and ECONOMICS
25-27 September 2020

Berlin, Germany

worse (Laborde et al., 2020). All these led to a phenomenon, known as "panic buying", where consumers emptied the shelves in the supermarkets (Sima et al., 2020).

Since the market demand for agricultural commodities increased, consequently, the values of these stocks also increased and this effect is depicted in the present paper results. The results are of great importance since the economy could give more weight on the primary sector, and not on the tertiary sector (services). In this paper, using relevant econometric techniques, we capture the impact of the COVID-19 spread on two most important commodities from the agricultural sector, namely the Oats and the Wheat.

The present paper contributes to the literature in the following ways: (a) It is the first, to the best of our knowledge, that investigates the effect of the Covid-19 spread and the lockdown measures on the agriculture sector; (b) It is the first, to the best of our knowledge, that accounts for the Covid-19 pandemic in a financial framework, using global data on the spread of the pandemic; (c) it proposes an alternative approach to the examination of the economic effect of crises on the agricultural sector, based on a financial framework; and (d) it provides a robustness analysis of the findings based on out-of-sample forecasting accuracy measures.

The paper is structured as follows: Section 2 describes the methodology used, Section 3 presents the empirical results and finally, Section 4 concludes the paper.

\section{Methodology}

In order to econometrically investigate (non-)causality between the Covid-19 confirmed cases and the Cruise companies' stocks, we will make use of Granger (non-)causality tests and the state of the art step-by-step (non-) causality test, introduced by Dufour and Renault (1998) and extended by Dufour et al. (2006). In this context, following standard time series literature (Wooldridge 2013), before turning to non-causality testing, we examine the level of integration of the time series that enter our analysis using the Phillips-Perron (1988) unit root test. More specifically, the hypothesis tested for the Phillips - Perron test is that the time series does not have a unit root. In addition, in case of integrated of degree one time series, i.e. I(1), we also investigate the potential existence of long-run relationships among the variables, using the popular Johansen (1990) cointegration test, and the hypothesis tested is that the time series are not cointegrated. Finally, the optimal lag length of the time series variables was investigated using the Schwartz-Bayes information criterion (SBIC).

Additionally, in order to cross validate the fact that the Covid-19 confirmed cases are causal and thus have predictive ability on the major Cruise companies' stocks, we will also make use of forecasting strategies. In detail, using a vector autoregressive model as a baseline, we will investigate whether alternative specification that could also incorporate the information provided by Covid-19 confirmed cases as an exogenous 
2nd International Conference on Applied Research in BUSINESS, MANAGEMENT and ECONOMICS
25-27 September 2020

Berlin, Germany

variable in the same vector autoregressive model, outperforms the forecasting accuracy of the baseline model. To do so, three distinct measure of forecasting accuracy are used, namely the mean absolute error (MAE), the mean absolute percentage error (MAPE) and the root mean square forecasting error (RMSFE), to investigate the magnitude of the predictive power of Covid-19 spread on the biggest Cruise companies' stocks. Of course, in order to statistically assess the differences in forecasting accuracy between the baseline and the alternative models, Mariano and Diebold (1995) tests are also implemented.

In what follows, we offer a brief outline of the techniques and procedures used in this work.

\subsection{Relevant tests}

Phillips-Perron unit root test:

As a first step, we check for the potential existence of unit roots in our time series using relevant unit root tests. More analytically, we implement the Phillips-Perron unit root test. The null hypothesis of the test, is that the time-series contain a unit root.

Johansen co-integration test:

In case of $\mathrm{I}(1)$ variables, we test for cointegration among the time-series. If cointegrating relationships are present, Error Correction Terms (ECM) have to be included in the model. In this work, we implement the Johansen (1988) test. The trace test, tests the null hypothesis of $r<n$ cointegrating vectors, while the maximum eigenvalue test, similarly, tests the null hypothesis of $r<r+1$ cointegrating vectors and the critical values are found in Johansen and Juselius (1990).

2.2 Causality testing between Covid-19 spread and Cruise companies' stocks

As a next step, we investigate (non-)causality between the Covid-19 confirmed cases and the Cruise companies' stocks, using the Granger (non-)causality tests.

\section{Granger Non-causality}

After examining the stationarity and cointegration properties of the data series, we use (non-)Granger causality to test the predictive ability of the variables that enter the model. More precisely, the hypothesis tested for Granger (non-)causality test is that the times series are not Granger causal, i.e. the logarithmic Covid-19 confirmed cases do not have predictive ability on the biggest cruise stocks. As Engle and Granger (1987) showed, if two variables are cointegrated, an Error Correction Model (ECM) should be employed in the model in order to capture the long-run relationship among the variables.

The ECM Granger non-causality test involves fitting the model:

$$
\Delta y_{t}=a_{0}+\sum_{i=1}^{m} a_{1 i} \Delta y_{t-i}+\sum_{i=0}^{m} a_{2 i} \Delta x_{t-i}+\lambda \mu_{t-1}+\varepsilon_{t}(\mathbf{1})
$$




\section{2nd International Conference on Applied Research in BUSINESS, MANAGEMENT and ECONOMICS}

\section{5-27 September 2020 \\ Berlin, Germany}

where $\Delta$ is the first difference operator, $\Delta \mathrm{y}_{\mathrm{t}}$ and $\Delta \mathrm{x}_{\mathrm{t}}$ are stationary time series and $\varepsilon_{\mathrm{t}}$ is the white noise error term with zero mean and constant variance. Also, $\mu_{\mathrm{t}-1}$ is the lagged value of the error term of the cointegration regression.

Next, in order to study the exact timing pattern of the causality relationship, we make use of the state-of-the-art step-by-step causality introduced by Dufour and Renault (1998) and extended by Dufour et al. (2006).

\section{Step-by-step causality}

Based on recent advancement of the related literature of causality, Granger non-causality tests fail to unveil the potential timing pattern of a causal relationship. In this context, in a seminal paper in Econometrica, Dufour and Renault (1998) introduced the notion of step-by-step or short-run causality based on the idea that two time series $\mathrm{X}_{\mathrm{t}}$ and $\mathrm{Y}_{\mathrm{t}}$ could interact in a causal scheme via a third variable $Z_{t}$. More precisely, despite the fact that $X_{t}$ could not cause $Y_{t}$ one period ahead, it could cause $Z_{t}$ one period ahead i.e. $Z_{t+1}$, and $Z t$ could cause $Y t$ two periods ahead i.e. $Y_{t+2}$. Therefore, $X_{t} \rightarrow Y_{t+2}$, even though $X_{t} \rightarrow Y_{t+1}$.

For testing the step by-step causality, consider the following VAR (p) model:

$$
Y_{t}=a+\sum_{k=1}^{p} \pi_{\kappa} Y_{t-k}+\sum_{q=0}^{Q} \beta_{q} X_{t-q}+u_{t}(\mathbf{3})
$$

where: $Y_{t}$ is an $(1 \mathrm{xm})$ vector of variables, a is a $(1 \mathrm{xm})$ vector of constant terms; $X_{t}$ is a vector of variables and $\mathrm{u}_{\mathrm{t}}$ is a $(1 \mathrm{xm})$ vector of error terms such that $E\left(u_{t} u_{s}\right)=\sigma_{i i}$ if $t=$ $s$ and $E\left(u_{t} u_{s}\right)=\sigma_{i j} I$ if $t \neq s$, where $I$ is the identity matrix. The lags in the baseline model are selected using the Schwartz-Bayes Information criterion (SBIC).

Following Dufour et al. (2006), the model described in (3) corresponds to horizon $h=1$. In order to test for the existence of non-causality in horizon $h$, the algorithm follows Konstantakis and Michaelides (2015).

\subsection{Econometric models}

A basic and classical econometric approach is the vector autoregressive model - VAR, or the vector error correction model - VECM in presence of co-integration relationship among the variables. In presence of co-integration relationship among the variables, we incorporate the error correction term.

Each variable in the model, has an equation explaining its evolution based on its own lagged values, the lagged values of the other model variables, the error correction, and an error term. A VECM model of order $\mathrm{p}$, with exogenous variables is structured as follows: 


$$
\left\{\begin{array}{c}
\Delta y_{1, t}=c_{1}+\sum_{j}^{n} \sum_{i}^{p}\left(a_{j, i} \Delta y_{j, t-i}\right)+\sum_{j}^{k} \sum_{i}^{q}\left(b_{j, i} \Delta x_{j, t-i}\right)+v_{1, t}+e_{1, t} \\
\Delta y_{2, t}=c_{2}+\sum_{j}^{n} \sum_{i}^{p}\left(a_{j, i} \Delta y_{j, t-i}\right)+\sum_{j}^{k} \sum_{i}^{q}\left(b_{j, i} \Delta x_{j, t-i}\right)+v_{2, t}+e_{2, t} \\
\cdots \\
\Delta y_{n, t}=c_{n}+\sum_{j}^{n} \sum_{i}^{p}\left(a_{j, i} \Delta y_{j, t-i}\right)+\sum_{j}^{k} \sum_{i}^{q}\left(b_{j, i} \Delta x_{j, t-i}\right)+v_{n, t}+e_{n, t}
\end{array}\right\}
$$

$\mathrm{n}$ is the number of the dependent variables $\left(y_{i, t}\right)$ of the model, $c_{i}$ is the fixed term, $\mathrm{p}$ is the lag order of the dependent variables, $e_{i, t}$ is the error term of each equation of the model, and $v_{i, t}$ is the error correction term, as before. In the case of exogenous variables, $\mathrm{k}$ is the number of the independent or exogenous variables $\left(x_{i, t}\right)$ of the model and $\mathrm{q}$ is the lag order of the exogenous variables.

\subsection{Information criterion}

In this paper, we make use of the so-called Schwartz-Bayes Information criterion (SBIC) introduced by Schwarz (1978), because it is an optimal selection criterion when used in finite samples (Breiman and Freedman, 1983; Speed and Yu, 1992). The SBIC is derived:

$$
\hat{k}=\operatorname{argmin}_{k \leq n}\left\{-2 \frac{\ln (L L(k))}{n}+k \frac{\ln (n)}{n}\right\}
$$

where $\mathrm{LL}(\mathrm{k})$ is the log-likelihood function of a VAR(k) model, $\mathrm{n}$ is the number of observations and $\mathrm{k}$ is the number of lags and $\hat{k}$ is the optimum lag length selected.

\subsection{Forecasting accuracy}

In the present paper, we make use of the forecasting accuracy measures: mean absolute error (MAE), the mean absolute percentage error (MAPE) and root mean square forecast error (RMSFE). In general, the smaller the values of each forecasting criterion, the better the forecasting value.

MAE

A model's MAE for forecast horizon $\mathrm{H}$ is given by the following:

$$
M A E=\frac{1}{H} \sum_{t=0}^{H}\left|F_{t}-A_{t}\right|(4.17)
$$

where: $h$ is the forecast horizon of the model, $F_{t}$ are the out-of-sample forecasted values of the model, and $A_{t}$ are the actual values. However, one of the main disadvantages of the MAE is the fact that is has no standard scale and it is not as comparable as a percentage. To overcome this problem we will also base our analysis on MAPE.

\section{MAPE}

A model's MAPE is given by the expression:

$$
M A P E=\frac{100}{h} \sum_{t=0}^{h} \frac{\left|F_{t}-A_{t}\right|}{\left|A_{t}\right|}
$$




\section{RMFSE}

The RMSFE is used to measure the forecasting error distribution. It is given by the expression:

$$
R M S F E=\sqrt{E\left[\left(Y_{T+1}-\hat{Y}_{T+1 \mid T}\right)^{2}\right]}
$$

\subsection{Forecast accuracy comparison}

In order to compare the forecasting accuracy of the baseline and alternative models, we use the Diebold - Mariano test. This test was established by Diebold and Mariano (1991), for the comparison of two forecast results.

First, we define the actual values: $\left\{y_{t}, t=1, \ldots, T\right\}$ and two forecast values: $\left\{\hat{y}_{1 t}, t=1, \ldots, T\right\}$ and $\left\{\hat{y}_{2 t}, t=1, \ldots, T\right\}$.

Next, we define the forecast errors as:

And,

$$
e_{i t}=\hat{y}_{i t}-y_{t}, i=1,2 \text { (2.29) }
$$

$$
g\left(e_{i t}\right)=\exp \left\{l e_{i t}\right\}-1-l e_{i t}(2.30)
$$

Function $\mathrm{g}$ is a loss function and 1 is a positive constant.

The loss differential between the two forecasts is:

$$
d_{t}=g\left(e_{1 t}\right)-g\left(e_{2 t}\right)(2.31)
$$

The null hypothesis of the Diebold - Mariano test is the following:

$$
H_{0}: E\left(d_{t}\right)=0
$$

And the respective alternative hypothesis:

$$
H_{0}: E\left(d_{t}\right) \neq 0
$$

\section{Result Analysis}

\subsection{Data and variables}

The data used in the present paper are the global Confirmed cases of the Covid-19 in daily format and were downloaded by the John Hopkins University database and span the period 22 January 2020 until 2 June 2020. Moreover, in the present paper we used the two major commodities from the agricultural sector, namely the Oats and Wheat, derived from finance.yahoo, and span also the period 22 January 2020 until 2 June 2020. The confirmed cases were transformed into logarithmic scale. The descriptive statistics of the timeseries are depicted in Table 1. Furthermore, the plots of the logarithmic confirmed Covid-19 cases and the two logarithmic values of the commodities are depicted on Figures 1-2. 
Table 1: Descriptive statistics of the timeseries.

Variable

Log_Confirmed Covid-19

cases

Log_Oats

Log_Wheat
Standard

Mean Deviation Min Max

2.7290
1.0203

2.7443

6.8047

0.0304

2.4035

2.5179

0.0183

2.7638

Figure 1\&2: Log Confirmed Covid-19 cases vs log values of Oats and Log Confirmed Covid-19 cases vs log values of Wheat.
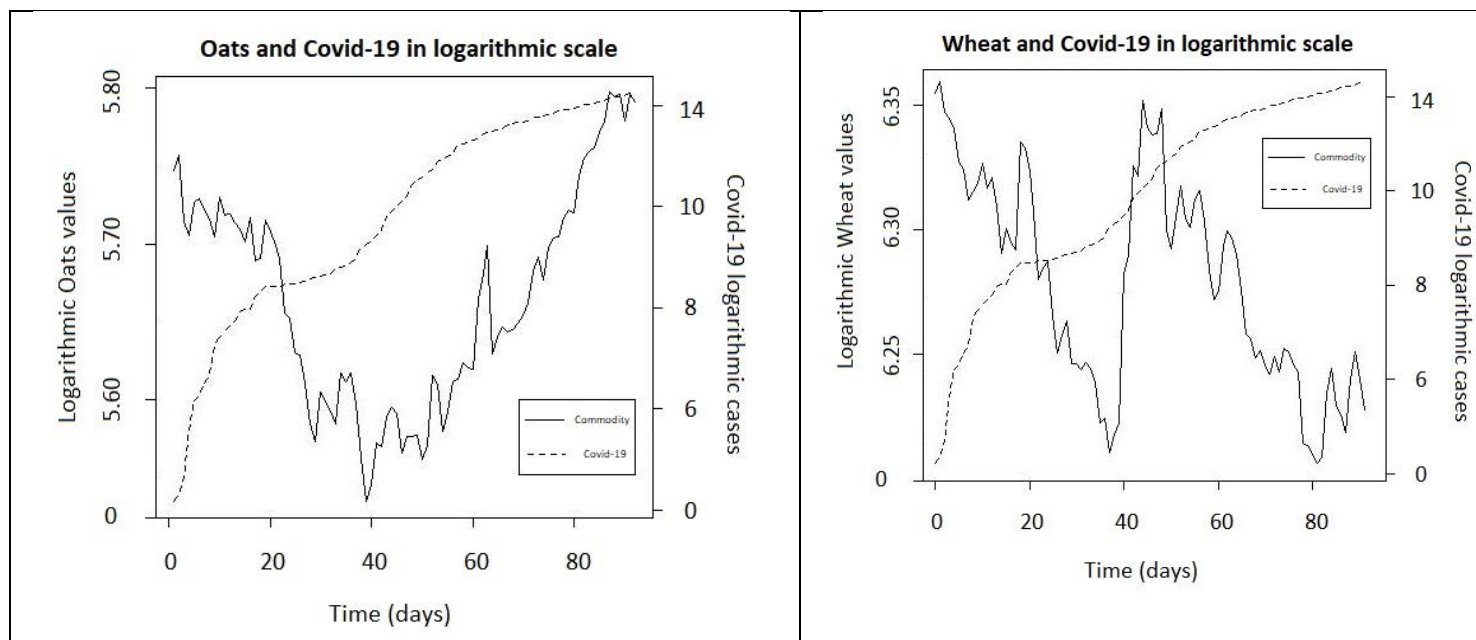

Figures 1-2 indicate that the logarithmic confirmed Covid-19 cases seem to have a lagged effect on the logarithmic values of the commodities. This fact provide a graphical evidence about the impact of the Covid-19 spread on the agricultural sector, a fact that needs to be investigated thoroughly using econometric methodology.

\subsection{Results}

In the present paper, we set out a hypothesis tested, that the Covid-19 spread, and more analytically, the current economic crisis and the lockdown measures following the Covid19 , affect the prices of the two commodities.

In the present work, we make use of the granger and step-by-step causalities in order to identify whether the Covid-19 spread causes the values of the two commodities. As a next step, we test the contribution of the information derived by the Covid-19 confirmed cases on the forecasting of the aforementioned commodities. To do so, we make use of two econometric models in the form of vector autoregressive model (since 
there is connection among the three stocks). The first model, declared as he baseline, will be a vector autoregressive model only with endogenous variables (the two commodity values) and an alternative model being the same with the baseline, augmented with one exogenous variable, the Covid-19 global confirmed cases. The comparison of these two models, in terms of forecasting ability, will unveil a possible contribution of the exogenous variable on the forecasting of the two commodity values.

A first step in every econometric modeling is the unit root test. In the present paper, we make use of the Phillips - Perron unit root test. The results are depicted in Table 2.

Table 2: Phillips - Perron unit root test results.

Variable PP test P-

Log_Confirmed Covid-19

$\begin{array}{lll}\text { cases } & 0.0100 & \mathrm{I}(0) \\ \text { Log_Oats } & 0.9230 & \mathrm{I}(1) \\ \text { Log_Wheat } & 0.3930 & \mathrm{I}(1)\end{array}$

Since the results in Table 2 show that the Covid-19 has no unit root, meaning that the timeseries is stationary, this specific timeseries cannot be cointegrated and thus, in the couple comparisons (Covid-19 and each commodity) we may infer that no cointegration relationships exist. However, in the case of the full model (all variables) we must test the presence of co-integration.

Table 3: Johansen Cointegration test results.

\begin{tabular}{lrrrl} 
Rank & test & 10 pct & \multicolumn{1}{l}{5 pct } & 1 pct \\
$\mathrm{r}<=2$ & 0.0400 & 6.5000 & 8.1800 & 11.6500 \\
$\mathrm{r}<=1$ & 9.0400 & 12.9100 & 14.9000 & 19.1900 \\
$\mathrm{r}=0$ & 26.6400 & 18.9000 & 21.0700 & 25.7500
\end{tabular}

The results of the co-integration test are depicted in Table 3, indicate that there is 1 cointegration relationship among the timeseries. In such case, an error correction term must be included in the econometric models.

The next step is the use of the causality tests, namely the Granger causality and the step-by-step causality. To start with, we give the results of the Granger causality (Table 4).

Table 4: Granger causality results for the case of the Oats and Wheat commodities. 


\begin{tabular}{lrr}
\hline Timeseries & P-value & \multicolumn{1}{c}{ Order } \\
\hline Oats & 0.0306 & 1 \\
Wheat & 0.0384 & 15 \\
\hline
\end{tabular}

The results in Tables 4 indicate that the Covid-19 causes the values of the two commodities. In order to identify the specific order, we make use of the step-by-step causality. The results are depicted in Tables 6 and 7, respectively.

Table 5: Step-by-step causality results for the case of the Oats and Wheat commodities.

\begin{tabular}{lll}
\hline Timeseries & P-values & Orders \\
\hline Oats & $0.006,0.0325,0.0553,0.1022$ & $2,3,4,7$ \\
Wheat & $0.0755,0.1025,0.0858,0.0509,0.041,0.0848$ & $8,9,15,16,17,18$ \\
\hline
\end{tabular}

The results in Table 5 indicate that the Covid-19 causes the commodities in multiple orders. The fact that these order differ in the step-by-step approach for the two commodities, shows that the Covid-19 causes, or in general, impacts these two commodities in a different manner.

Since we have shown that the Covid-19 spread causes the values of the two commodities, and therefore it provides useful information for their interpretation and their modeling, we will test if the Covid-19 spread contributes to their forecasting. To do so, we first decide for the lag order of the econometric models (baseline and alternative), based on the SBIC criterion. The results are depicted in Table 6 .

Table 6: Results of the SBIC criterion for the case of the Baseline and Alternative models.

\begin{tabular}{rrr}
\hline Order & Baseline model & Alternative model \\
\hline 1 & -16.2582 & -21.7664 \\
2 & -16.0888 & -21.4130 \\
3 & -15.8957 & -21.0244 \\
4 & -15.7091 & -20.6223 \\
5 & -15.5798 & -20.3185 \\
6 & -15.3839 & -20.3536 \\
7 & -15.1995 & -19.9320 \\
8 & -15.0024 & -19.5475 \\
9 & -14.8113 & -19.3755 \\
10 & -14.6043 & -18.9846 \\
\hline
\end{tabular}


Based on Table 6, the SBIC criterion indicate the lag order 1 as the most appropriate for both models. In this case, the order of the baseline and alternative order will be selected to be equal to 1 .

The baseline model incorporated one lag order for each endogenous variables (Oats, Wheat). Using out of sample forecast with fixed window, for horizon $\mathrm{H}=1,2, \ldots, 10$, we forecast for two weeks, based on the business calendar. Then, we employ the same model incorporating as exogenous variable the logarithmic global confirmed Covid-19 cases and test again the forecasting ability of this alternative model. We then compare the two models in terms of forecasting ability, based on the MAE, MAPE, RMSFE and estimate the statistical significance of the comparison based on Diebold-Mariano test. The results are depicted in Tables 7 and 8 .

Table 7: MAE, MAPE and RSMFE forecasting accuracy of the VEC and VECX models for the case of Oats.

\begin{tabular}{|c|c|c|c|c|c|c|}
\hline $\begin{array}{l}\text { Horiz } \\
\text { on }\end{array}$ & $\begin{array}{l}\text { MAE_V } \\
\text { EC }\end{array}$ & $\begin{array}{l}\text { MAPE_V } \\
\text { EC }\end{array}$ & $\begin{array}{l}\text { RMSFE_V } \\
\text { EC }\end{array}$ & $\begin{array}{l}\text { MAE_VE } \\
\text { CX }\end{array}$ & $\begin{array}{l}\text { MAPE_VE } \\
\text { CX }\end{array}$ & $\begin{array}{l}\text { RMSFE_VE } \\
\text { CX }\end{array}$ \\
\hline 1 & 0.0073 & 0.0013 & 0.0073 & 0.0009 & 0.0002 & 0.0009 \\
\hline 2 & 0.0105 & 0.0018 & 0.0109 & 0.0005 & 0.0001 & 0.0006 \\
\hline 3 & 0.0159 & 0.0028 & 0.0179 & 0.0023 & 0.0004 & 0.0034 \\
\hline 4 & 0.0213 & 0.0037 & 0.0244 & 0.0041 & 0.0007 & 0.0056 \\
\hline 5 & 0.0292 & 0.0051 & 0.0348 & 0.0083 & 0.0014 & 0.0123 \\
\hline 6 & 0.0346 & 0.0060 & 0.0405 & 0.0100 & 0.0017 & 0.0136 \\
\hline 7 & 0.0392 & 0.0068 & 0.0453 & 0.0110 & 0.0019 & 0.0141 \\
\hline 8 & 0.0411 & 0.0071 & 0.0465 & 0.0101 & 0.0017 & 0.0133 \\
\hline 9 & 0.0449 & 0.0078 & 0.0505 & 0.0101 & 0.0017 & 0.0130 \\
\hline 10 & 0.0479 & 0.0083 & 0.0534 & 0.0093 & 0.0016 & 0.0123 \\
\hline
\end{tabular}

Table 8: MAE, MAPE and RSMFE forecasting accuracy of the VEC and VECX models for the case of Wheat.

\begin{tabular}{|c|c|c|c|c|c|c|}
\hline $\begin{array}{l}\text { Horiz } \\
\text { on }\end{array}$ & $\begin{array}{l}\text { MAE_V } \\
\text { EC }\end{array}$ & $\begin{array}{l}\text { MAPE_V } \\
\text { EC }\end{array}$ & $\begin{array}{l}\text { RMSFE_V } \\
\text { EC }\end{array}$ & $\begin{array}{l}\text { MAE_VE } \\
\text { CX }\end{array}$ & $\begin{array}{l}\text { MAPE_VE } \\
\text { CX }\end{array}$ & $\begin{array}{l}\text { RMSFE_VE } \\
\text { CX }\end{array}$ \\
\hline 1 & 0.0073 & 0.0012 & 0.0073 & 0.0072 & 0.0012 & 0.0072 \\
\hline 2 & 0.0234 & 0.0038 & 0.0284 & 0.0231 & 0.0037 & 0.0280 \\
\hline 3 & 0.0342 & 0.0055 & 0.0398 & 0.0336 & 0.0054 & 0.0390 \\
\hline 4 & 0.0377 & 0.0060 & 0.0420 & 0.0368 & 0.0059 & 0.0410 \\
\hline 5 & 0.0403 & 0.0065 & 0.0439 & 0.0393 & 0.0063 & 0.0427 \\
\hline 6 & 0.0421 & 0.0067 & 0.0451 & 0.0409 & 0.0066 & 0.0438 \\
\hline 7 & 0.0474 & 0.0076 & 0.0514 & 0.0461 & 0.0074 & 0.0500 \\
\hline
\end{tabular}


2nd International Conference on Applied Research in BUSINESS, MANAGEMENT and ECONOMICS
25-27 September 2020

Berlin, Germany

0.0577

0.0624

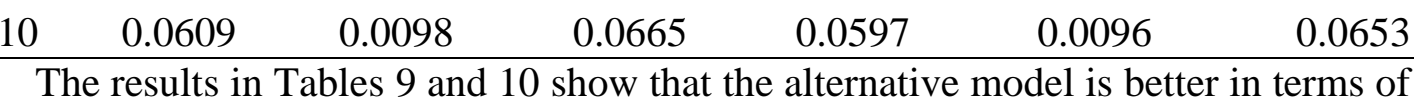
forecasting ability than the baseline, for the two commodities (Oats and Wheat). All results, based on the Diebold-Mariano test are statistical significant (except for the first horizon in the case of Wheat). This implies that the Covid-19 has augments the forecasting ability of the values of the two commodities.

Finally, using the impulse - response function, and more precisely, the orthogonalized impulse responses, the results indicate that the effect of the Covid-19 on the Oats commodity, is statistically significant, and positive, as depicted in Figure 3. This means that a unit shock in the Covid-19 spread, will lead to a positive effect in the Oats commodity. In the same context, the effect of the Covid-19 on the Wheat commodity, is statistically significant, and positive, only in the start of the shock, as depicted in Figure 4. This means that a unit shock in the Covid-19 spread, will lead to a positive effect in the Wheat commodity.

Figure $3 \& 4$ : Impulse response function for the case of Oats and the case of Wheat.

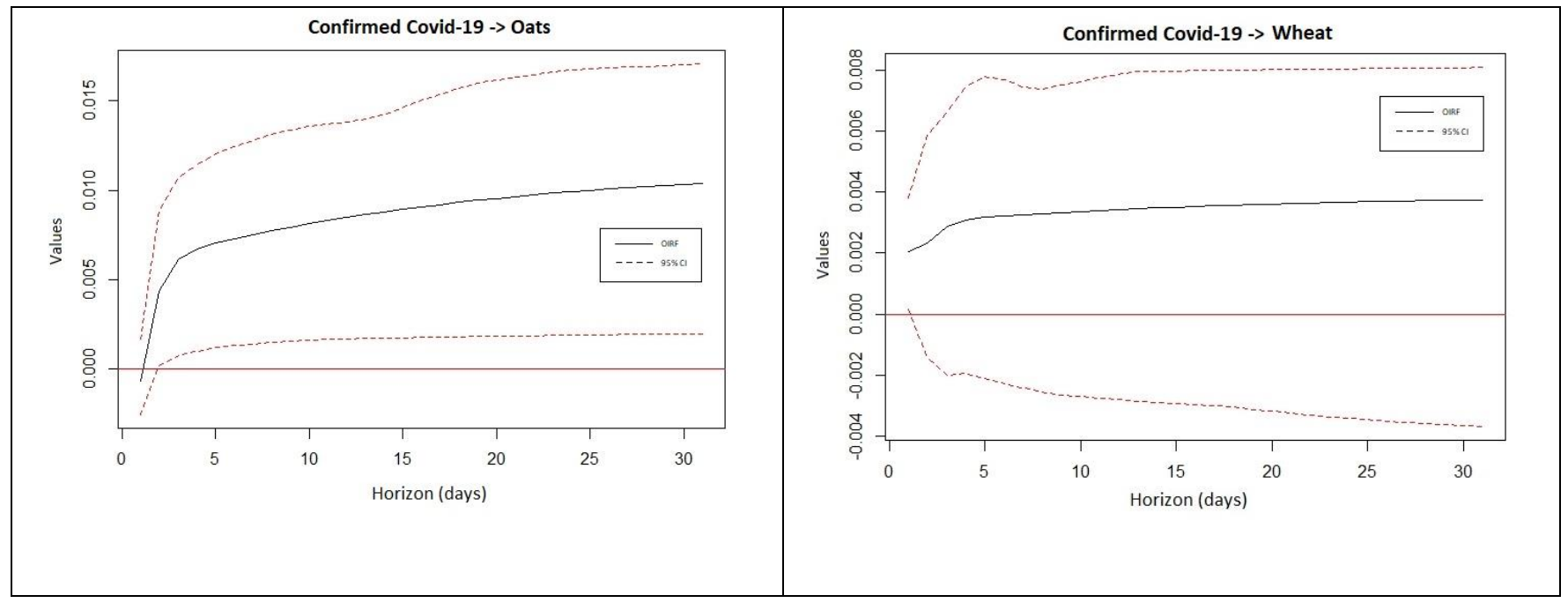

\section{Conclusion}

In the present paper, we examine two most important agriculture commodities, namely, the wheat and oats commodities during the Covid-19 spread and lockdown measures. Using relevant time series specifications, we establish a hypothesis regarding the effect of the Covid-19 on the prices of these commodities. The hypothesis is proved, as, based on our findings, the Covid-19 spread, Granger causes and also step-by-step 
2nd International Conference on Applied Research in BUSINESS, MANAGEMENT and ECONOMICS
25-27 September 2020

Berlin, Germany

causes the wheat and oats commodities. These results indicate that the Covid-19 spread provide useful information for the modeling of these commodities as it affects them, econometrically speaking.

Furthermore, the Covid-19 spread provides useful information for the forecasting of these commodities, as shown by the forecasting comparison of the baseline and alternative models, indicated by the forecasting criteria MAE, MAPE and RMSFE, with statistical significance of the comparison, as shown by the Diebold-Mariano test. Our findings are robust, since the out-of-sample forecasting accuracy of the alternative model employed, that explicitly incorporate the pandemic induced by the SARS-COV-2 virus, are superior to the baseline model.

The present paper findings imply that the Covid-19 spread not only contributes with statistically significant information in the modeling of the two most important agricultural commodities, but also increase the forecasting ability of these commodities in the 22/10 - 02/06 time period of the year 2020. The results give credit to the impact of the Covid-19 spread on the Agriculture. This fact unveils the great impact of the Covid19 on the agricultural industry worldwide, affecting the overall economy.

We anticipate our work to be a starting point for more sophisticated models, testing for other factors that could play a significant role in forecasting the various agricultural commodities. Clearly, future and more extended research on the subject would be of great interest.

\section{References}

Breiman L.A. and Freedman D.F. (1983), How many variables should be entered in a regression equation?, Journal of American Statistical Association, 78, pp. 131-136.

Diebold X. Francis and Mariano S. Roberto (1991), Comparing predictive accuracy I: An asymptotic test, Discussion paper 52, Federal reserve bank of Minneapolis, Institute for empirical macroeconomics, , pp. 1-29.

Dufour J.-M. and Pelletier D. and Renault E. (2006), Short run and Long run Causality in Time series: Inference, Journal of Econometrics, 132 (2): 337-362.

Dufour, J.-M., Renault, E. (1998), Short-run and long-run causality in time series: theory, Econometrica, 66 (5): 1099-1125.

Engle, F.R. and Granger, W.J.C. (1987), Co-integration and error correction: representation, estimation, and testing, Econometrica 55 (2): 251-276. 
Ganesan Kanagavalli and Micheal Manida (2020), Covid-19:Impact of agriculture in India, Aegaeum, ISSN NO: 0776-3808, https://www.researchgate.net/publication/341324024.

Ganesan Kanagavalli and Micheal Manida (2020), Covid-19: Impact of agriculture in India, Aegaeum, ISSN NO: 0776-3808, https://www.researchgate.net/publication/341324024.

Johansen Søren (1988), Statistical analysis of cointegration vectors, Journal of Economic Dynamics and Control, Volume 12, Issues 2-3, Pages 231-254.

Johansen Soren, Juselius Katarina (1990), Maximum likelihood estimation and inference on cointegration-with applications to the demand for money, Oxford Bulletin of Economics and Statistics, 52(2): 169-210.

Laborde David, Mamun Abdullah, Parent Marie (2020), Documentation for the COVID19 Food Trade Policy Tracker: Tracking Government Responses Affecting Global Food Markets during the COVID-19 Crisis, https://www.ifpri.org/project/covid-19-food-tradepolicy-tracker.

Phillips Peter C. B. and Perron Pierre (1988), Testing for a Unit Root in Time Series Regression, Biometrika, Vol. 75, No. 2, pp. 335-346.

Schwarz G.E. (1978), Estimating the dimension of a model, Annals of Statistics, 6 (2): 461-464.

Sima Kang, Chuaa Hong Choon, Vietab Eduard and Fernandeza George (2020), The anatomy of panic buying related to the current COVID-19 pandemic, Psychiatry Research, 288, 113015.

Speed T.P and Yu Bin (1992), Model selection and prediction: Normal Regression, Annals of the Institute of Statistical Mathematics, Vol. 45, Issue 1, pp. 35-54.

Wooldridge J. M. (2013), Introductory Econometrics: A Modern Approach. 5th ed. Mason. OH: South-Western. 